\title{
Pratiques phytosanitaires et niveau d'exposition aux pesticides des producteurs de coton du nord du Bénin
}

\author{
Abdoul-Ibrachi Gouda ${ }^{1,2,{ }^{*}}$, Ibrahim Imorou Toko ${ }^{1}$, Sharaf-Dine Salami ${ }^{1}$, Maïté Richert ${ }^{2}$, \\ Marie-Louise Scippo ${ }^{3}$, Patrick Kestemont ${ }^{4}$ et Bruno Schiffers ${ }^{2}$ \\ ${ }^{1}$ Laboratoire de recherche en aquaculture et écotoxicologie aquatique (LaRAEAq), département des sciences et techniques de production \\ animale et halieutique (STPAH), faculté d'agronomie, université de Parakou, Parakou, Bénin \\ ${ }^{2}$ Gembloux Agro-Bio Tech/université de Liège (ULiège), laboratoire de phytopharmacie, Liège, Belgique \\ ${ }^{3}$ Laboratoire d'analyse des denrées alimentaires, FARAH-Santé publique vétérinaire, faculté de médecine vétérinaire/université de Liège \\ (ULiège), Liège, Belgique \\ ${ }^{4}$ Unité de recherche en biologie environnementale et évolutive (URBE), faculté des sciences, université de Namur, Namur, Belgique
}

\begin{abstract}
Résumé - Au Bénin, les écosystèmes terrestres sont pollués par la présence généralisée de résidus de pesticides due à une utilisation intensive de produits phytosanitaires en agriculture, principalement sur la culture du coton. Afin d'évaluer le niveau d'exposition des producteurs aux pesticides et d'estimer l'impact potentiel de ceux-ci sur la santé humaine, des enquêtes semi-structurées couplées à des observations sur les pratiques locales ont été effectuées auprès de 150 producteurs de coton des communes de Gogounou, Kandi et Banikoara, principales zones de production cotonnière du pays. Soixante-quinze pour cent des producteurs interrogés n'ont jamais bénéficié d'une scolarisation, et seulement $5 \%$ d'entre eux ont été formés à l'utilisation sans danger des pesticides sur la culture du coton. Parmi les pesticides utilisés par les producteurs, seuls $19 \%$ appartiennent à la liste des produits homologués au Bénin. Les substances actives les plus fréquemment utilisées sont des insecticides tels que l'acétamipride, la lambda-cyhalothrine, le chlorpyrifos-éthyle, l'émamectine benzoate, le profénofos ou la cyperméthrine. Toutes ces substances sont connues pour être toxiques et pourraient avoir des effets néfastes sur la santé après une exposition. Soixantequinze pour cent des producteurs interrogés utilisent des doses d'insecticide supérieures à celles recommandées sur les étiquettes et $80 \%$ ne portent pas d'équipement de protection individuelle, que ce soit lors de la préparation de la bouillie, du chargement ou de la pulvérisation. Les emballages vides de pesticides sont souvent abandonnés dans les champs de coton ( $73 \%$ des observations) ou parfois utilisés à des fins domestiques ( $25 \%$ des observations). Les observations de terrain traduites en scénarios fiables ont permis d'estimer les niveaux d'exposition des producteurs à l'aide du modèle prédictif UK-POEM. Les expositions totales sans équipement de protection individuelle varient de 0,099 à $0,546 \mathrm{mg} / \mathrm{kg}$ de poids corporel/jour. Ces valeurs d'exposition dépassent largement les niveaux d'exposition acceptables pour l'opérateur, indiquant un risque potentiel.
\end{abstract}

Mots clés : coton / pesticide / évaluation de risque / UK-POEM / Bénin

\begin{abstract}
Plant protection practices and pesticide exposure levels of Northern Benin cotton producers. In Benin, terrestrial ecosystems are polluted by a widespread presence of pesticide residues released by an intensive use of plant protection products in agriculture, mainly those sprayed on cotton crops. In order to assess the exposure of producers to pesticides and predict the potential impact on human health, field observation of local practices coupled with semi-structured surveys were conducted among 150 cotton growers in Gogounou, Kandi and Banikoara in Northern Benin, the main cotton production area of the country. Seventy-five percent of producers never received an education nor instructions of use, while only $5 \%$ have been trained in the safe use of pesticides on cotton crops. Among pesticides used by farmers, only $19 \%$ belong to the approved list of plant protection products in Benin. The most frequently used active substances are insecticides such as acetamiprid, lambda-cyhalothrin, chlorpyrifos-ethyl, emamectin benzoate, profenofos or cypermethrin. All are known to be more or less toxic and may have detrimental
\end{abstract}

\footnotetext{
$\overline{\text { *Auteur de correspondance }}$ : goudaibrachi@yahoo.fr
} 
effects on health after exposure. Seventy-five percent of producers use higher amounts of insecticides than recommended on the labels and $80 \%$ do not wear personal protective equipment during mixing, loading and spraying. Empty pesticide containers are often left in cotton fields ( $73 \%$ of observations) or sometimes used for domestic purposes (25\% of observations). Field observations were translated into reliable scenarios to estimate the exposure levels of producers, using the UK-POEM predictive model. Total exposures without personal protective equipment vary from 0.099 to $0.546 \mathrm{mg} / \mathrm{kg}$ body weight/day. Exposure values far exceed the Acceptable Operator Exposure Levels, indicating a potential risk.

Keywords: cotton / pesticide / risk assessment / UK-POEM / Benin

\section{Introduction}

Au Bénin, la culture du coton consomme à elle seule près de $96 \%$ des engrais chimiques utilisés (IFDC, 2007) et près de $90 \%$ des insecticides (Ton, 2001). En effet, en raison du programme stratégique de relance du secteur agricole, qui vise l'amélioration des rendements, on assiste à une utilisation accrue de pesticides en agriculture pour lutter contre les ravageurs, les maladies et les adventices. Selon Oerke et Dehne (1997), sans un contrôle des adventices et des ravageurs par les pesticides, environ $42 \%$ de la production agricole mondiale serait perdue chaque année. Au Bénin, Agbohessi et al. (2011) dénoncent l'utilisation frauduleuse, par les producteurs de coton, de molécules phytosanitaires d'origine et de qualité diverses. Cependant, nonobstant l'efficacité des pesticides sur les ravageurs et leur effet positif sur l'augmentation des rendements, plusieurs études au Bénin (Ahouangninou et al., 2011; Agbohessi et al., 2012), au Togo (Kanda, 2011) et au Burkina Faso (Lehmann et al., 2016) ont montré que l'utilisation répétée et mal maîtrisée de produits chimiques pour la lutte phytosanitaire n'est pas sans conséquences pour la santé des agriculteurs, des consommateurs ou pour l'environnement. Ainsi, à cause de l'usage intensif des pesticides, différents écosystèmes africains (terrestres et aquatiques) sont contaminés par des résidus (Okoumassoun et al., 2002 ; Traoré et al., 2006; Lehmann et al., 2016; Son et al., 2017). Cela s'explique par le fait que seulement $0,1 \%$ des pesticides pulvérisés dans les champs atteignent leur cible, le reste se dispersant dans le milieu en contaminant l'air, la terre et l'eau (Pimentel, 1995).

Cette situation paraît inquiétante dans la mesure où l'on assiste aujourd'hui à la promotion de la filière coton au Bénin, considérée comme prioritaire au détriment de toutes les autres cultures. Cette filière utilise à chaque saison culturale des milliers de litres de pesticides stockés puis pulvérisés par les producteurs, sans un minimum de précautions pour éviter les risques de contamination.

C'est dans ce contexte qu'une étude a été conduite dans le bassin cotonnier du nord du Bénin, auprès des producteurs de coton et des structures de gestion de la filière, pour analyser les pratiques phytosanitaires en vigueur, évaluer l'exposition potentielle des producteurs aux produits phytopharmaceutiques utilisés dans les conditions habituelles et évaluer leurs impacts potentiels sur la santé humaine. Elle s'inscrit dans le cadre du projet ARES-AQUATOX (Bénin), qui a pour objectif d'attirer l'attention des producteurs et des autorités sur les risques associés à l'usage incontrôlé des pesticides et sur la nécessité de respecter de meilleures pratiques phytosanitaires, notamment pour éviter la contamination potentielle des nombreuses retenues d'eau présentes à proximité des champs et utilisées pour la production et la fourniture d'eau de boisson.

\section{Méthodologie}

\subsection{Choix de la zone d'étude}

L'étude a été conduite dans le bassin cotonnier du Bénin situé dans le département de l'Alibori (Fig. 1). Trois communes (Banikoara, Kandi et Gogounou) ont été retenues à la fois pour leur forte utilisation de pesticides au cours de la saison culturale, pour leurs potentialités piscicoles et pour la proximité des retenues d'eau avec des champs de coton. Il s'agit des trois premières communes en termes de production de coton (Agbohessi et al., 2011). Dans chaque commune, les deux villages qui possédaient les plus grandes retenues d'eau ont été choisis pour réaliser les enquêtes de terrain (Tab. 1). En effet, de mauvaises pratiques phytosanitaires engendrent non seulement des risques sanitaires directs (exposition des opérateurs), mais aussi indirects (contamination des ressources en eau destinées à l'alimentation des hommes et des animaux domestiques ou sauvages).

\subsection{Technique de collecte des données}

L'étude a été conduite de 2014 à 2017 pendant la principale période de production du coton. La méthode de collecte des données est une enquête individuelle semi-structurée, réalisée auprès de 150 producteurs de coton ayant au moins cinq années d'expérience dans la production de coton. Les producteurs interrogés ont été identifiés par la technique dite «boule de neige » et ont été retenus à partir d'un tirage aléatoire de leur numéro d'enregistrement dans la Coopérative villageoise des producteurs de coton de chaque village. L'observation directe et participative a été utilisée pour suivre les producteurs lors des phases de préparation de la bouillie et de pulvérisation des cotonniers sur le terrain.

\subsection{Données collectées}

Les données collectées concernent les différents produits phytosanitaires utilisés, les substances actives qu'ils contiennent, les circuits d'approvisionnement, la toxicité et autres propriétés reprises dans les bases de données disponibles sur les sites www.agritox.anses.fr, www.echa.europa.eu/fr/ regulations/clp/classification (Classification, Labelling and Packaging of substances and mixtures) et www.sagepestici des.qc.ca (AGRITOX, 2018; ECHA, 2018; SAgE PESTICIDES, 2018). La liste des pesticides utilisés a été complétée grâce 


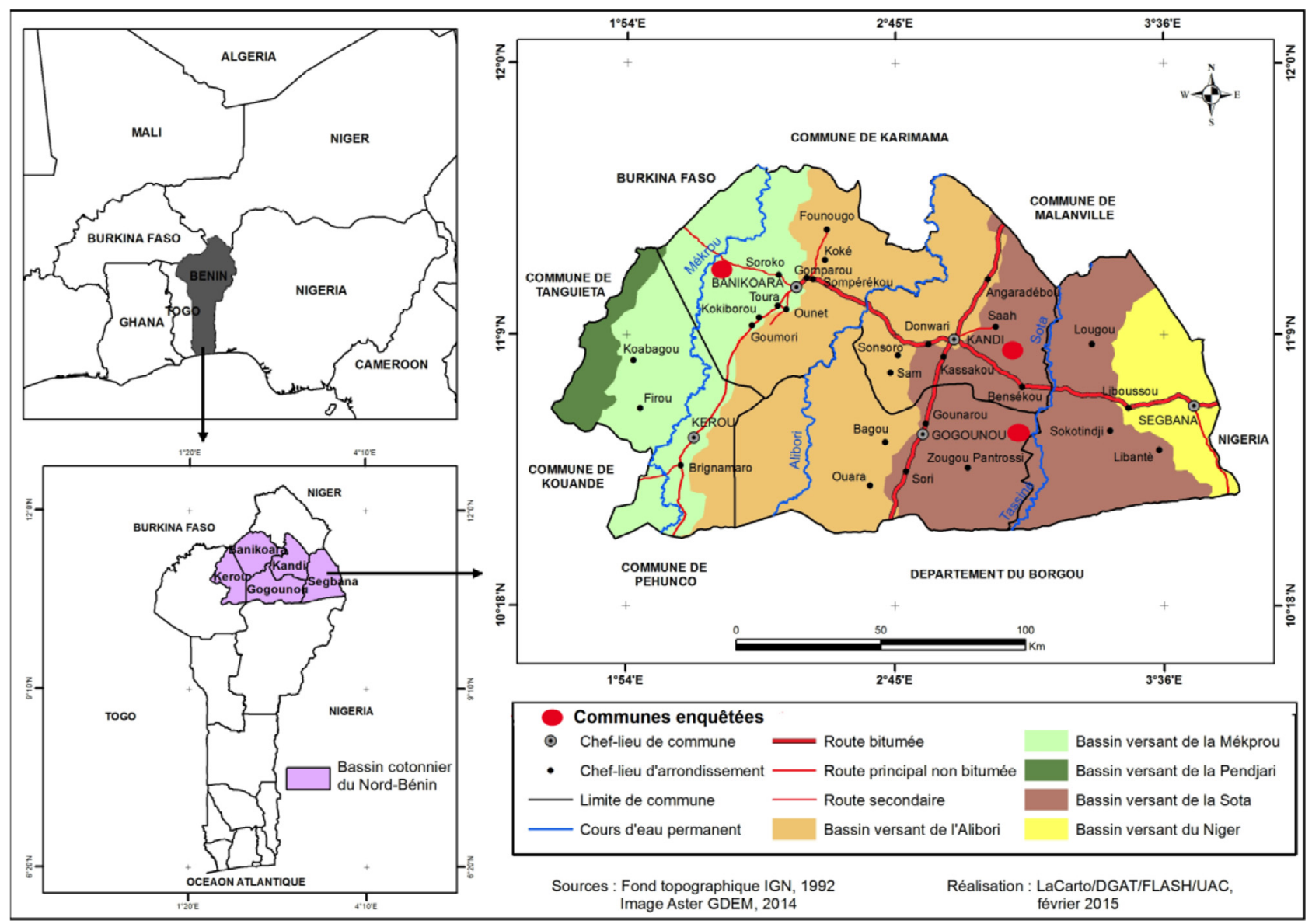

Fig. 1. Zone d'étude: le bassin cotonnier au Bénin.

Fig. 1. Study area: the cotton basin in Benin.

Tableau 1. Consommation officielle de pesticides sur les cultures de coton et superficie des retenues d'eau dans les trois communes sélectionnées.

Table 1. Official pesticide consumption for cotton crops and water reservoirs surface in each surveyed municipality.

\begin{tabular}{llcclc}
\hline \multirow{2}{*}{ Commune } & \multicolumn{2}{c}{ Quantité de pesticides utilisés (L) } & & \multirow{2}{*}{ Villages sélectionnés / Nom des retenues } & Superficie des retenues d'eau (ha) \\
\cline { 2 - 4 } & Campagne & Herbicide & Insecticide & & 16,0 \\
\hline \multirow{2}{*}{ Banikoara } & $2013-2014$ & 169870 & 133227 & Batran & 3,2 \\
& $2014-2015$ & 336561 & 145657 & Ounet & 1,5 \\
Kandi & $2013-2014$ & 79793 & 125893 & Gambanè & 2,4 \\
& $2014-2015$ & 215029 & 90063 & Sinawongounou peuhl & 7,7 \\
Gogounou & $2013-2014$ & 50319 & 47943 & Kérou-Bagou & 2,3 \\
\hline
\end{tabular}

Source: données de la SONAPRA de 2015.

à l'examen des emballages abandonnés sur le terrain et des produits vendus sur les marchés locaux. Les observations ont particulièrement porté sur la préparation des bouillies, qui est une phase majeure de risque pour les utilisateurs, car c'est le moment où ils sont en contact avec des produits concentrés. Les données collectées ont été saisies et traitées à l'aide des tableurs Excel (2013) et Statview 1.7 (1997).

\subsection{Modèle utilisé pour évaluer l'exposition potentielle des producteurs}

Pour estimer le niveau d'exposition potentielle des producteurs sur une journée de travail (en $\mathrm{mg} / \mathrm{kg}$ de poids corporel/jour), le modèle britannique «Predictive Operator Exposure Model»(UK-POEM) a été utilisé car il intègre la 
Tableau 2. Classement par ordre décroissant de l'exposition aux pesticides des producteurs de coton (mg/kg de poids corporel/jour). Table 2. Decreasing ranking of pesticide exposure of cotton producers ( $\mathrm{mg} / \mathrm{kg}$ body weight/day).

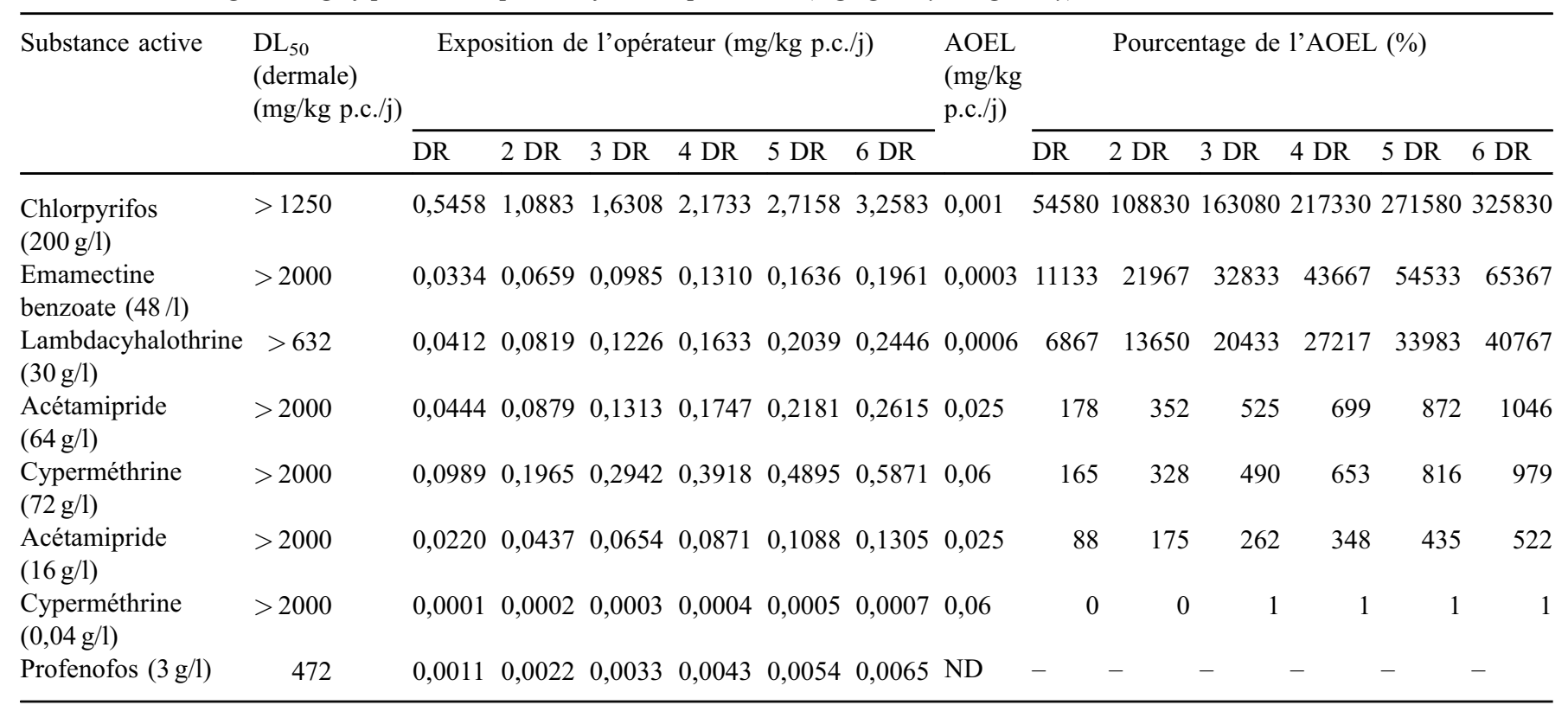

$\mathrm{AOEL}=$ niveau d'exposition acceptable pour l'opérateur $; \mathrm{DL}_{50}=$ dose létale $50 ; \mathrm{ND}=$ non disponible $; \mathrm{DR}=$ dose recommandée $; n$ DR $=n$ fois la dose recommandée (avec $n=2,3,4,5,6) ; \mathrm{mg} / \mathrm{kg}$ p.c. $/ \mathrm{j}=$ milligramme par kilogramme de poids corporel par jour.

possibilité de choisir de petits appareils d'épandage. Il a été utilisé pour la même raison par plusieurs auteurs (Illyassou et al., 2017; Lawson et al., 2017; Son et al., 2017) pour évaluer l'exposition des petits producteurs qui manipulent les produits phytosanitaires. Les paramètres observés tels que la méthode d'application, la formulation et la concentration de la substance active, l'équipement de protection individuelle porté, ou encore la dose et le volume d'application, ont été entrés dans le modèle UK-POEM. Le niveau d'exposition a été évalué pour chacune des substances actives appliquées sur la culture (valeurs par défaut dans le modèle: $6 \mathrm{~h}$ et 1 ha traité) durant tout le cycle végétatif (6 traitements phytosanitaires). Un scénario d'exposition sans équipement de protection individuelle a été considéré, dans la mesure où la majorité des agriculteurs $(80 \%)$ ne se protègent pas lors des traitements phytosanitaires. Les valeurs d'exposition prédictives, pour les opérations de mélangechargement et lors de la pulvérisation, ont été évaluées, avec une absorption dermale de $10 \%$ et inhalatoire de $100 \%$, pour 7 substances actives identifiées dans les insecticides liquides les plus utilisés. Les valeurs toxicologiques $\left(\mathrm{DL}_{50}\right.$ dermale) et les seuils limites acceptables ou AOEL (Acceptable Operator Exposure Level) de chaque substance active ont été obtenus dans la base de données de la Commission européenne (EU Pesticides Database, 2018) et sont présentés dans le tableau 2. Enfin, le risque théorique présenté par chaque substance a été caractérisé en comparant l'exposition systémique totale estimée (en $\mathrm{mg} / \mathrm{kg}$ de poids corporel/jour) aux valeurs d'AOEL.

Les données météorologiques ont été enregistrées pendant chaque pulvérisation. Elles sont restées dans la fourchette recommandée pour la pulvérisation du coton (Schiffers, 2011; Salah et al., 2015). Un thermo-hygromètre (TFA, Kat. Nr.30.5007) et un anémomètre (IHM CFM/CMM 6190 SI) ont servi à l'enregistrement de ces données.

\section{Résultats}

\subsection{Caractéristiques sociodémographiques des producteurs de coton}

Les producteurs de coton rencontrés et enquêtés sont en majorité des hommes (94\%) âgés de 25 à 66 ans. Soixante-quinze pour cent d'entre eux n'ont reçu aucune instruction, $17 \%$ ont atteint le niveau primaire et seulement $5 \%$ ont reçu il y a dix ans une formation sur les traitements phytosanitaires du cotonnier (Tab.3). Les traitements phytosanitaires en production cotonnière sont exclusivement réalisés par des hommes. Les femmes productrices de coton ( $6 \%$ de notre échantillon) se font aider par leur époux ou leurs fils.

\subsection{Diversité des pesticides et effets des substances actives}

Un total de 78 produits commerciaux a été répertorié, dont 37 insecticides et 41 herbicides. Parmi ceux-ci, seulement $19 \%$ sont homologués au Bénin (CNAC, 2012). Les 6 insecticides les plus utilisés par les producteurs dans le bassin cotonnier du Bénin sont par ordre d'importance: Killerselect $^{(8)}(80 \%)$, Cutter $(71 \%)$, Moacartarine 215 $(55 \%)$, Chémapride $88(34 \%)$, Sharp Shooter $(27 \%)$ et Spider 315 (12\%) (les substances actives et les doses préconisées sont indiquées dans le Tab. 4). Selon leur classification CLP (Classification, Labelling and Packaging of substances and mixtures), règlement (CE) $\mathrm{n}^{\mathrm{o}} 1272 / 2008$, ces substances actives sont toxiques pour l'homme et dangereuses pour l'environnement aquatique. 
Tableau 3. Statut social des producteurs de coton dans le bassin cotonnier du nord du Bénin.

Table 3. Social status of cotton growers in the cotton basin of Northern Benin.

\begin{tabular}{lc}
\hline Caractéristiques des producteurs & $\begin{array}{l}\text { Pourcentage (\%) } \\
\text { du total }\end{array}$ \\
\hline Sexe & 94 \\
Homme & 6 \\
Femme & \\
Niveau d'instruction & 76 \\
Aucun & 17 \\
Primaire & 7 \\
Secondaire & \\
Nombre de formations reçues & \\
sur les traitements phytosanitaires & \\
et la gestion des emballages & 95 \\
Aucune & 5 \\
$\geq 1$ & \\
\hline
\end{tabular}

\subsection{Gestion des produits phytosanitaires dans le bassin cotonnier}

\subsubsection{Circuit d'approvisionnement en produits phytosanitaires}

Trois grands circuits d'approvisionnement des produits phytosanitaires ont été identifiés dans le bassin cotonnier du Bénin. Le premier est un circuit formel de distribution et de commercialisation, régi par les textes en vigueur au Bénin sur les produits phytosanitaires (décret n ${ }^{\circ}$ 92-258 de 1992 et arrêté $\mathrm{n}^{\mathrm{o}}$ 255/MDR/MCAT/DC/CC/CP de 1993). Tous les produits commercialisés reçoivent l'agrément du Comité national d'homologation, d'agrément et de contrôle des pesticides. Dans notre enquête, tous les producteurs ont obtenu leurs produits phytopharmaceutiques, notamment les insecticides, à travers ce circuit. Néanmoins, il existe aussi un circuit informel, approvisionné par les produits venant des pays frontaliers (Ghana, Nigéria, Togo, Burkina Faso). Environ $40 \%$ des producteurs de notre échantillon se ravitaillent également sur le marché local, surtout en herbicides. Enfin, un troisième circuit est généré par les producteurs de coton euxmêmes, qui revendent les produits acquis dans le circuit formel; trente pour cent des producteurs de notre échantillon ont reconnu se livrer à ce «trafic».

\subsubsection{Doses d'insecticides appliquées par les producteurs de coton}

Le nombre moyen de traitements insecticides recommandés par cycle de production du coton (selon le programme de traitement phytosanitaire du cotonnier) est de six, à raison d'une fois par quinzaine à partir du $45^{\mathrm{e}}$ jour après la levée, avec une dose de 0,51/ha (soit 2 flacons à l'hectare pour l'insecticide Killerselect ${ }^{\circledR}$ ). Les doses répertoriées en fonction du type d'insecticide varient entre 2 et 6 flacons à l'hectare et $85 \%$ des producteurs réalisent des traitements avec une dose d'insecticide supérieure à celle recommandée pour les insecticides (Tab. 5). Il ressort des résultats que les producteurs de coton utilisent de fortes doses d'insecticides (bien supérieures à la dose recommandée), y compris aux environs immédiats des retenues d'eau.

\subsubsection{Gestion des emballages vides}

Les emballages vides sont souvent abandonnés dans la nature, dans la plupart des cas dans les champs de coton (73\%), et sont dans certains cas utilisés à des fins domestiques $(25 \%)$, pour le stockage de l'huile pour la cuisine, comme récipient pour transporter la bouillie ou l'eau de boisson des enfants qui vont au champ ou à l'école. Rarement (2\%), ils sont incinérés ou enfouis dans le sol.

\subsubsection{Affections ressenties par les producteurs après l'application de pesticides}

Quatre-vingt pour cent des producteurs ne se protègent pas lors de l'application des produits phytosanitaires dans les champs de coton. La majorité des producteurs interrogés $(85 \%)$ reconnaissent avoir ressenti, après utilisation des pesticides, des effets qu'ils attribuent aux traitements effectués et qui se manifesteraient de différentes façons: irritations cutanées $(60 \%$ des cas déclarés), toux $(13 \%)$, céphalées $(11 \%)$, nausées $(8 \%)$, affections oculaires $(7 \%)$, et même vertiges lorsque le producteur, par imprudence, reçoit une dose de produit importante entraînée par le vent au niveau du visage (Fig. 2).

\subsection{5 Évaluation des risques}

Les résultats de l'enquête montrent que les producteurs utilisent fréquemment des doses d'insecticides de 2 à 6 fois supérieures à celles qui sont recommandées. Le niveau d'exposition a été évalué pour les différentes doses utilisées (Tab. 2).

\section{Discussion}

\subsection{Utilisation des pesticides dans le bassin cotonnier}

Dans le bassin cotonnier du Bénin, la forte pression parasitaire, le faible taux d'instruction et le manque de formation des producteurs de coton constituent les principaux facteurs conduisant à une utilisation abusive des produits phytosanitaires, comme l'ont montré d'autres études menées au Bénin (Ahouangninou et al., 2011), au Burkina Faso (Son et al., 2017), en Côte d'Ivoire (Wognin et al., 2013), au Sénégal (Badiane, 2004) ou au Togo (Kanda et al., 2013).

Au cours des cinq dernières années, les pyréthrinoïdes, les néonicotinoïdes et l'avermectine ont été les familles d'insecticides les plus utilisées contre les ennemis du cotonnier, dont Helicoverpa armigera (Hübner). Cependant, plusieurs auteurs ont montré l'inefficacité des pyréthrinoïdes vis-à-vis de cette chenille (Houndété et al., 2010; Gnankiné et al., 2013). Cette situation amène les producteurs non seulement à augmenter le nombre de traitements et les doses (Son et al., 2017), mais aussi à douter de l'efficacité des produits recommandés par les 


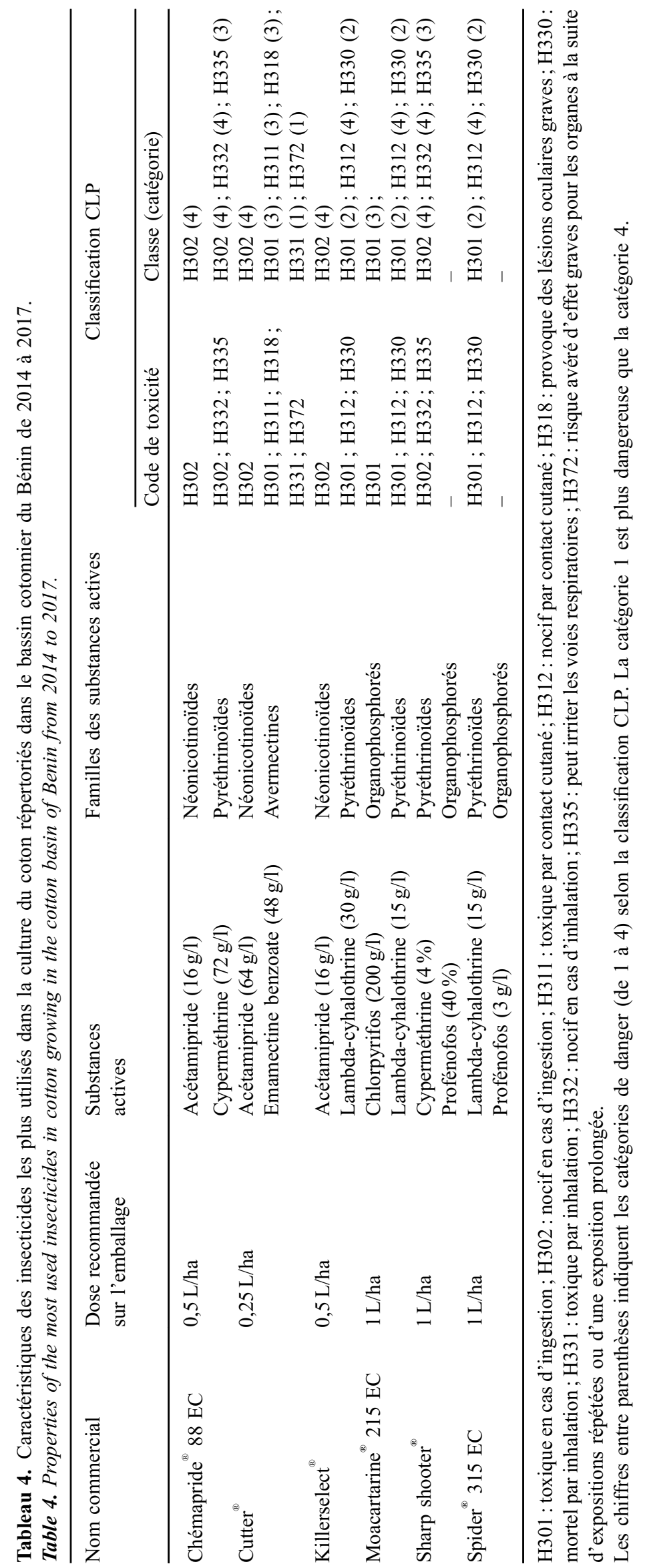

Page 6 de 9 
Tableau 5. Dose d'insecticide (Killerselect ${ }^{\circledR}$ ) utilisée par les producteurs de coton.

Table 5. Dose of insecticide (Killerselect) used by cotton producers.

\begin{tabular}{lccc}
\hline $\begin{array}{l}\text { Dose d'insecticide utilisée } \\
\text { à l'hectare (flacons/ha) }\end{array}$ & $\begin{array}{l}\text { Nombre de producteurs } \\
\text { de coton }\end{array}$ & $\begin{array}{l}\text { Proportion des } \\
\text { producteurs }(\%)\end{array}$ & $\begin{array}{l}\text { Observations } \\
2\end{array}$ \\
\hline 23 & 15,3 & $\begin{array}{l}\text { Dose recommandée/ha } \\
\text { Dosage supérieur à } \\
\text { celui recommandé/ha }\end{array}$ \\
4 & 26 & 17,3 & 34,7 \\
5 & 52 & 21,3 & 11,3 \\
6 & 32 & 100,0 & \\
Total & 17 & & \\
\hline
\end{tabular}

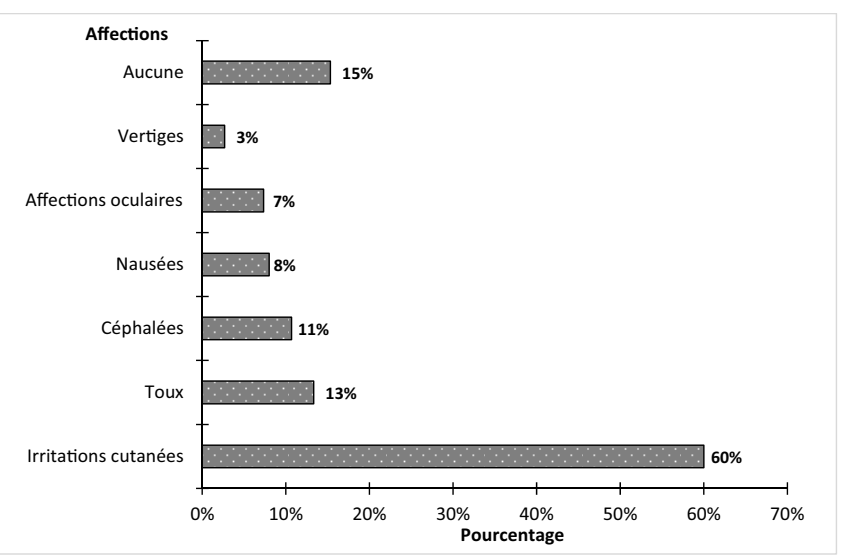

Fig. 2. Affections ressenties par les producteurs interrogés après l'application de pesticides.

Fig. 2. Affections experienced by interviewed producers after pesticide application.

services d'encadrement. Ils ont alors tendance à recourir à d'autres produits phytosanitaires, dont l'efficacité est meilleure, mais l'origine douteuse. Plusieurs auteurs dénoncent l'existence de circuits d'approvisionnement informels au Bénin (Adechian et al., 2015). Cette situation existe aussi au Burkina Faso (Gomgnimbou et al., 2009; Son et al., 2017).

Le stockage des produits phytosanitaires se fait sans respect des normes conventionnelles de l'Organisation des Nations unies pour le développement industriel (ONUDI) et, après les traitements, les emballages vides sont abandonnés dans l'environnement ou utilisés à des fins domestiques. Ces résultats corroborent ceux de Doumbia et Kwadjo (2009) en Côte d'Ivoire, de Kanda et al. (2013) au Togo ou de Son et al. (2017) au Burkina Faso, qui ont tous souligné la mauvaise gestion des stocks et des emballages vides de produits phytosanitaires. Pour Gomgnimbou et al. (2009), l'utilisation des boîtes vides de pesticides, après ou sans lavage, pour conditionner les eaux de consommation est une source de danger sanitaire pour les populations.

L'exposition des opérateurs aux produits phytosanitaires varie selon les conditions d'application. Plusieurs auteurs ont montré l'importance de la température et de l'humidité de l'air, qui affectent, d'une part, la volatilité du produit (Schiffers et Moreira, 2011; El-Aissaoui, 2015), et, d'autre part, le taux de transpiration qui favorise une pénétration plus rapide du produit dans le corps (Aubertot et al., 2005; Gil et al., 2008). Cependant, il a été observé que, dans le bassin cotonnier du Bénin, les applications sont habituellement effectuées lorsque les conditions météorologiques sont favorables (températures fraîches et vitesse du vent dans la plage recommandée de 1 à $2 \mathrm{~m} / \mathrm{s}$ ) (Schiffers, 2011; Salah et al., 2015).

\subsection{Mauvaises pratiques phytosanitaires}

La classification selon le règlement $(\mathrm{CE}) \mathrm{n}^{\circ} 1272 / 2008$, dit CLP, montre que la quasi-totalité des substances actives identifiées sur les emballages sont toxiques ou nocives en cas d'ingestion et par contact cutané. Plusieurs études ont déjà souligné l'existence de mauvaises pratiques lors de l'utilisation des pesticides (Adechian et al., 2015; Son et al., 2017).

Le risque d'intoxication est accru quand les doses sont élevées et que la protection est inexistante. L'étude montre que $85 \%$ des producteurs utilisent des doses d'insecticides plus élevées que celles recommandées, sans se protéger. Les mêmes constats ont été faits par Duemmler (1993) et Guissou et al. (1996), qui estiment que l'exposition aux insecticides des producteurs de coton lors des traitements, pendant une longue durée et sans matériel de protection adéquat, constitue une source majeure de risque pour leur santé. Le risque d'exposition pourrait être considérablement réduit si les agriculteurs portaient un équipement de protection individuelle complet. En effet, selon Toé et al. (2013) et Richard et al. (2014), les équipements de protection individuelle jouent un rôle très important dans la réduction de l'exposition des opérateurs aux produits phytosanitaires. Les producteurs béninois, compte tenu de leur faible niveau d'instruction et d'encadrement, méconnaissent la toxicité des pesticides utilisés et maîtrisent peu leur utilisation. Les conséquences de cette situation sont l'intoxication aiguë et chronique des agriculteurs, mais aussi l'exposition des consommateurs aux résidus de pesticides, qui est sous-estimée (Ahouangninou et al., 2011; Ngom et al., 2012; Lehmann et al., 2016).

\section{3 Évaluation des niveaux d'exposition des producteurs de coton}

Les résultats de l'estimation du niveau d'exposition des producteurs de coton montrent que ceux-ci sont très exposés 
aux produits phytosanitaires dans le bassin cotonnier du Bénin (les pourcentages de l'AOEL fournis par le modèle varient entre 165 et $54580 \%$ ). Plus les doses utilisées sont élevées, plus cette tendance se renforce. Ainsi, pour l'acétamipride, à la dose recommandée sans équipement de protection individuelle, l'exposition est de $88 \%$ de l'AOEL. Mais en doublant la dose recommandée, l'exposition passe à $175 \%$ de l'AOEL. Les risques sont critiques, par ordre d'importance, pour le chlorpyrifos-éthyl, l'émamectine benzoate, la lambda-cyhalothrine, l'acétamipride et la cyperméthrine. Ils font partie des substances les plus utilisées ces dernières années au Bénin, non seulement sur la culture du coton (Agbohessi et al., 2011; Adechian et al., 2015), mais aussi sur les cultures maraîchères (Ahouangninou, 2013).

Les malaises ressentis par $85 \%$ des producteurs interrogés (irritations cutanées, toux, céphalées, nausées et vertiges) pourraient s'expliquer par le caractère nocif ou toxique des substances actives. Les mêmes affections ont été rapportées par Gomgnimbou et al. (2009) ou Son et al. (2017) sur des producteurs de coton et de cultures maraîchères au Burkina Faso. La santé des producteurs est ainsi menacée du fait de l'absence du port d'équipements de protection individuelle lors de la préparation et de l'exécution des traitements phytosanitaires. Pourtant, selon Nicourt et Girault (2009), les producteurs ne s'exprimeraient pas facilement sur la relation entre santé et pesticides. Ils estiment développer une immunité à l'égard de ces produits depuis des années. Or, les produits utilisés, inhalés même à des doses inférieures à celles utilisées au Bénin, ont des effets significatifs sur la santé (Richard et al., 2005).

\section{Conclusion}

Dans le bassin cotonnier au Bénin, plusieurs substances actives nocives ou toxiques ont été identifiées sur les marchés locaux, sur des emballages vides présents chez les producteurs ou abandonnés dans les champs. Dans leur majorité, les producteurs de coton reconnaissent la dangerosité des produits, parfois en ressentent les effets, mais ils en ignorent les risques à long terme pour leur santé, celle des autres consommateurs ou pour leur environnement. L'approvisionnement des producteurs en produits phytosanitaires à partir du circuit informel, le non-respect des doses recommandées lors des traitements phytosanitaires, l'utilisation de pesticides toxiques et non recommandés, l'absence d'utilisation d'équipement de protection individuelle, l'utilisation des emballages vides à des fins domestiques, constituent autant de pratiques qui exposent dangereusement les populations béninoises du bassin cotonnier. Face à ces pratiques qui prennent de l'ampleur, les structures d'encadrement techniques sont interpellées pour former les producteurs, améliorer leurs connaissances sur l'importance des équipements de protection, la gestion des emballages vides et l'emplacement des champs de coton par rapport aux retenues d'eau, ainsi que sur la notion de risque.

Remerciements. Les auteurs tiennent à remercier l'ARES pour le soutien financier au projet de recherche AQUATOX.

\section{Références}

Adechian AS, Baco MN, Akponikpe I, Toko II, Egah J, Affoukou K. 2015. Les pratiques paysannes de gestion des pesticides sur le maïs et le coton dans le bassin cotonnier du Bénin, VertigO - La revue électronique en sciences de l'environnement 15(2). DOI: 10.4000/ vertigo.16534.

Agbohessi TP, Imorou Toko I, Yabi AJ, Dassoundo CFJ, Kestemont P. 2011. Caractérisation des pesticides chimiques utilisés en production cotonnière et impacts sur les indicateurs économiques dans la commune de Banikoara au nord du Bénin. International Journal of Biological and Chemical Sciences 5(5): 1828-1841.

Agbohessi TP, Imorou Toko I, Kestemont P. 2012. État des lieux de la contamination des écosystèmes aquatiques par les pesticides organochlorés dans le bassin cotonnier béninois. Cahiers Agricultures 21: 46-56. DOI: 10.1684/agr.2012.0535.

AGRITOX. 2018. Base de données sur les substances actives phytopharmaceutiques. Disponible sur http://www.agritox.anses. $\mathrm{fr} /$, consulté le 13/06/2018.

Ahouangninou CCA. 2013. Durabilité de la production maraîchère au sud-bénin: un essai de l'approche écosystémique. Thèse de doctorat en Gestion de l'environnement, Université d'AbomeyCalavi, Bénin, 349 p. Disponible sur http://agritrop.cirad.fr/ 572410/.

Ahouangninou C, Fayomi BE, Martin T. 2011. Évaluation des risques sanitaires et environnementaux des pratiques phytosanitaires des producteurs maraîchers dans la commune rurale de Tori-Bossito (Sud-Bénin). Cahiers Agricultures 20(3): 216-222. DOI: 10.1684/ agr.2011.0485.

Aubertot JN, Barbier JM, Carpentier A, Gril JJ, Guichard L, Lucas P, et al. 2005. Pesticides, agriculture et environnement. Réduire l'utilisation des pesticides et limiter leurs impacts environnementaux. Expertise Scientifique Collective, INRA/CEMAGREF, 64 p.

Badiane M. 2004. Utilisation des pesticides dans le système maraîcher périurbain: variations annuelle et impacts sur la santé des populations. Thèse de doctorat en Médecine Vétérinaire, Dakar, EISMV, 125 p.

CNAC. 2012. Liste des produits phytopharmaceutiques sous autorisation provisoire de vente (APV) et agrément homologation (AH). Bénin, Porto-Novo: CNAC, 5 p.

Doumbia M, Kwadjo KE. 2009. Pratiques d'utilisation et de gestion des pesticides par les maraîchers en Côte d'Ivoire: cas de la ville d'Abidjan et deux de ses banlieues (Dabou et Anyama). Journal of Applied Biosciences 18: 992-1002.

Duemmler C. 1993. Pesticides et agriculture tropicale: dangers et alternatives. Paris: Dunod, $281 \mathrm{p}$.

ECHA. 2018. Classification des substances et des mélanges (European Chemicals Agency). Disponible sur www.echa. europa.eu/fr/regulations/clp/classification (19/06/2018).

El-Aissaoui A. 2015. Guide pratique pour les opérateurs agricoles: les bases de l'application rationnelle des produits phytosanitaires. Settat (Maroc): INRA, $32 \mathrm{p}$.

EU - Pesticides Database. 2018. European pesticides database. Available from http://ec.europa.eu/food/plant/pesticides/eu-pestici des-database/public/?event $=$ activesubstance. detail\&language $=$ EN\&selectedID=1755 (20/06/2018).

Gil Y, Sinfort C, Guillaume S, Brunet Y, Palagos B. 2008. Influence of micrometeorological factors on pesticide loss to the air during vine spraying: Data analysis with statistical and fuzzy inference models. Biosystems Engineering 100: 184-197. 
Gnankiné O, Mouton L, Savadogo A, Martin T, Sanon A, Dabire RK, et al. 2013. Biotype status and resistance to neonicotinoids and carbosulfan in Bemisia tabaci (Hemiptera: Aleyrodidae) in Burkina Faso, West Africa. International Journal of Pest Management 59 (2): 95-102. DOI: 10.1080/09670874.2013.771806.

Gomgnimbou APK, Savadogo PW, Nianogo AJ, Millogo-Rasolodimby J. 2009. Usage des intrants chimiques dans un agrosystème tropical : diagnostic du risque de pollution environnementale dans la région cotonnière de l'est du Burkina Faso. Biotechnologie, agronomie, société et environnement 13(4): 499-507.

Guissou IP, Toé MA, Domo Y, Hema OS. 1996. Contribution à la toxicologie agro-alimentaire au Burkina Faso : épidémiologie des intoxications aux pesticides et activités cholinestérasiques sériques chez les producteurs de la zone cotonnière de la boucle du Mouhoun. Études et recherches 4-5: 39-48.

Houndété TA, Kétoh GK, Hema OSA, Brévault T, Glitho IA, Martin T. 2010. Insecticide resistance in field populations of Bemisia tabaci (Hemiptera: Aleyrodidae) in West Africa. Pest Management Science 66: 1181-1185. DOI: 10.1002/ps.2008.

IFDC. 2007. Problématique de l'utilisation des produits phytosanitaires en conservation des denrées alimentaires et en maraîchage urbain et péri urbain au Burkina Faso: cas de Bobo Dioulasso, Ouahigouya et Ouagadougou. Muscle Shoals, USA: International Fertilizer Development Center (IDFC), 51 p. Available from http:// www.youscribe.com/BookReader/Index/539508?documentId= 510631 .

Illyassou KM, Adamou R, Schiffers B. 2017. Risk assessment for small farmers exposed to plant protection products in the Niger river valley. Communications in Agricultural and Applied Biological Sciences 81: 1-13.

Kanda M. 2011. Agriculture maraîchère au Togo: Analyse systémique et environnementale, Thèse de doctorat, Université de Lomé, Togo, 153 p.

Kanda M, Djaneye-Boundjou G, Wala K, Gnandi K, Batawila K, Sanni A, et al. 2013. Application des pesticides en agriculture maraîchère au Togo. VertigO - la revue électronique en sciences de l'environnement 13(1): 1-17. DOI: 10.4000/vertigo.13456.

Lawson AJ, Akohou H, Lorge S, Schiffers B. 2017. Three methods to assess levels of farmers' exposure to pesticides in the urban and peri-urban areas of Northern Benin. Tunisian Journal of Plant Protection 12: 91-108.

Lehmann E, Oltramare C, Nfon DJJ, Konaté Y, De Alencastro LF. 2016. Assessment of occupational exposure to pesticides with multi-class pesticide residues analysis in human hairs using a modified QuEChERS extraction method, case study of gardening areas in Burkina Faso. In: Annual Meeting of the International Association of Forensic Toxicologists (TIAFT), Brisbane, Australia.

Ngom S, Traoré S, Thiam MB, Anastasie M. 2012. Contamination des produits agricoles et de la nappe phréatique par les pesticides dans la zone des Niayes au Sénégal. Revue des sciences et de la technologie, Synthèse 25: 119-130.

Nicourt C, Girault JM. 2009. Le coût humain des pesticides: comment les viticulteurs et les techniciens viticoles français font face au risque, VertigO - la revue électronique en sciences de l'environnement 9(3) [En ligne]. DOI: 10.4000/vertigo.9197.

Oerke EC, Dehne HW. 1997. Global crop protection and the efficacity of crop protection, current situation and future trends. European Journal of Plant Pathology 103: 203-215.

Okoumassoun LE, Brochu C, DeBlois C, Akponan S, Marion M, Averill-Bates D, et al. 2002. Vitellogenin in tilapia male fishes exposed to organochlorine pesticides in Ouémé River in Republic of Benin. Science of the Total Environment 299: 163-172.

Pimentel D. 1995. Amounts of pesticides reaching target pests: environmental impacts and ethics. Journal of Agricultural and Environmental Ethics 8: 17-29.

Richard O, Toé AM, Ilboudo S, Guissou PI. 2014. Risk of workers exposure to pesticides during mixing/loading and supervision of the application in sugarcane cultivation in Burkina Faso. International Journal of Environmental Science and Toxicology Research 2(7): 143-151.

Richard SS, Moslemi H, Sipahutar N, Benachour, Seralini GE. 2005. Differential effects of glyphosate and roundup on human placental cells and aromatase. Environmental Health Perspectives 113(6): 716-720

SAgE PESTICIDES. 2018. Toxicité des substances actives. Disponible sur http://www.sagepesticides.qc.ca/Recherche/Recher cheMatiere (19/06/2018).

Salah SOT, De Cock N, Massinon M, Schiffers B, Dorbolo S, Lebeau F. 2015. Étude des potentialités des systèmes d'application contrôlée des gouttes (CDA) pour les traitements phytosanitaires en céréaliculture (synthèse bibliographique). Biotechnologie, agronomie, société et environnement 2016 20(S1): 287-298.

Schiffers B. 2011. Itinéraire technique de la tomate cerise (Lycopersicon esculentum). Bruxelles (Belgique): COLEACP/ PIP, $46 \mathrm{p}$.

Schiffers B, Moreira C. 2011. Fondements de la protection des cultures, Manuel $n^{\circ}$ 7. COLEACP/PIP Press. Bruxelles (Belgique) : Programme PIP/COLEACP, $294 \mathrm{p}$.

Son D, Somda I, Legreve A, Schiffers B. 2017. Pratiques phytosanitaires des producteurs de tomates du Burkina Faso et risques pour la santé et l'environnement. Cahiers Agricultures 26: 25005. DOI: $10.1051 /$ cagri/2017010.

Toé AM, Ouédraogo R, Ilboudo S, Guissou PI, 2013. Pilot study on agricultural pesticide poisoning in Burkina Faso. Interdiscip Toxicol 6(4): 185-191.

Ton P. 2001. Cotton production and rural livelihoods in West Africa. Situation and trends in the context of trade liberalisation and structural adjustment. African Environment, Occasional Papers, No. 219. Dakar (Senegal): Oxfam-GB \& ENDA-Tiers Monde, 87 p.

Traoré SK, Mamadou K, Dembélé A, Lafrance P, Mazelliert P, Houenou P. 2006. Contamination de l'eau souterraine par les pesticides en régions agricoles en Côte-d'Ivoire (Centre, Sud et Sud-Ouest). Journal africain des sciences de l'environnement 1: 1-9.

Wognin AS, Ouffoue SK, Assemand EF, Tano K, Koffi-Nevry R. 2013. Perception des risques sanitaires dans le maraîchage à Abidjan, Côte d'Ivoire. International Journal of Biological and Chemical Sciences 7(5): 1829-1827. DOI: 10.4314/ijbcs.v7i5.4.

Citation de l'article : Gouda A-I, Imorou Toko I, Salami S-D, Richert M, Scippo M-L, Kestemont P, Schiffers B. 2018. Pratiques phytosanitaires et niveau d'exposition aux pesticides des producteurs de coton du nord du Bénin. Cah. Agric. $27: 65002$. 